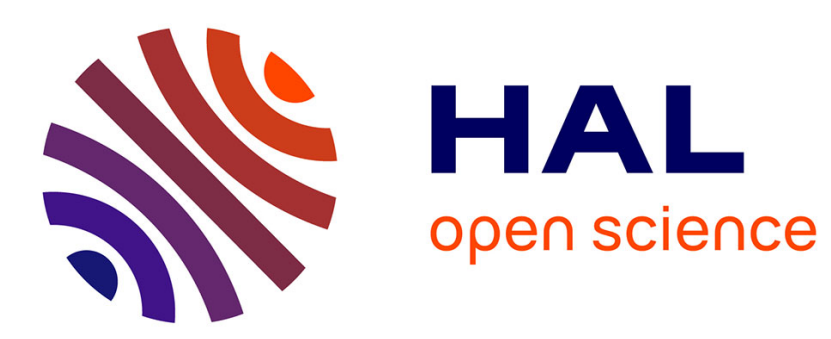

\title{
Impact of Wireless Medium Access Protocol on the Quadrotor Formation Control
}

Jose Alfredo Guerrero, Yacine Challal, Pedro Castillo

\section{To cite this version:}

Jose Alfredo Guerrero, Yacine Challal, Pedro Castillo. Impact of Wireless Medium Access Protocol on the Quadrotor Formation Control. Flight Formation Control, John Wiley, pp.257-276, 2012. hal00662820

\section{HAL Id: hal-00662820 \\ https://hal.science/hal-00662820}

Submitted on 25 Jan 2012

HAL is a multi-disciplinary open access archive for the deposit and dissemination of scientific research documents, whether they are published or not. The documents may come from teaching and research institutions in France or abroad, or from public or private research centers.
L'archive ouverte pluridisciplinaire HAL, est destinée au dépôt et à la diffusion de documents scientifiques de niveau recherche, publiés ou non, émanant des établissements d'enseignement et de recherche français ou étrangers, des laboratoires publics ou privés. 


\title{
Impact of Wireless Medium Access Protocol on the Quadrotor Formation Control
}

\author{
Jose Alfredo Guerrero and Yacine Challal and Pedro Castillo
}

\begin{abstract}
In this paper, the impact of the medium access protocols on the average consensus problem over wireless networks for a group of quadrotors is established. We study the case of a group of quadrotors communicating over a wireless network considering both directed and undirected graphs of information flow. It turns out that the media access control (MAC) protocols have a direct impact in both convergence time and average consensus solution, i.e. the solution of the average consensus is no longer the average of the initial conditions. It will be shown that the solution for the average consensus problem over a wireless network depends directly on the MAC algorithm. Simulations are provided to demonstrate the theoretical results.
\end{abstract}

\section{INTRODUCTION}

In recent years, much attention has been directed toward multiple robot systems with global or local communications, such as swarming [1], [2], flocking [3], [4], [5], [6], consensus [7] [8], [9], formations [10], [11], [12], etc. Different approaches have been proposed in the literature for coordination of multiple autonomous robot systems such as leader/follower [13], [14], virtual structure [15], [16] and behavioral control [17] and [18]. In the particular case of multi-agent (multiple mobile robot) consensus problem, most of the literature is focused on modeling the information flow network using either fixed or switching topologies. This means that the communication network does not suffer of time delay and packet drop problems. The main difference between fixed and switching topologies is that the switching topology case takes into account that every mobile robot has a limited range of interaction with its neighbors, i.e., the mobility of the robots affects the information exchange topology.

The distributed nature of multiple robot control system over wireless networks represent an interesting research problem. Data loss, data corruption and time delay over lossy network are key factors that may lead to performance degradation and even cause instability. Recent work on networked control over noisy communication channels includes [19], [20]. In most of the cases the packet drop phenomena is modeled as a random process without any specification of its probabilistic distribution [21], [22]. In [23], the authors consider the packet drop process as a Bernoulli process and develop stability conditions under these conditions. Another way to model the packet loss phenomena has been described

J. A. Guerrero, Y. Challal and P. Castillo are with the HEUDIASYC laboratory UMR 6599 CNRS-UTC, BP. 20529, 60200 Compiègne, France (jguerrer, ychallal, castillo) dhos.utc.fr. This work was supported by the SIRENE project. in [24], where a Markov chain has been used to model the packet dropout process.

This paper addresses the issue of packet loss as well as packet delay in a multi-agent aerial system considering a wireless network. For this end, we use the widely adopted network simulator (NS2) which provides a rich simulation environment modeling the different network communication layers: the physical layer (modulation, frequencies, signal and radio propagation models, wired and wireless channels, ...), link layer (different medium access control algorithms: TDMA, CSMA/CD, CSMA/CA, ALOHA, ...), routing layer (routing protocols over wired and wireless networks as well as mobile ad hoc networks), transport layer (TCP, UDP, RTP, etc.), and the application layer with a rich sample of applications for typical traffic generation scenarios. It will be assumed, for simplicity, that every agent in the multivehicle system broadcasts its information to its neighbors considering a fixed topology of information exchange, i.e. the mobility of the vehicles does not affect the information flow network. It is assumed that the neighbors of the $i^{t h}$. quadrotor are always in the $i^{t h}$-quadrotor broadcasting range. Different network media access control protocols have been designed for wireless networks. Likewise, in this paper, we analyze the impact of the following algorithms on the multiple quadrotor average consensus problem: Carrier sense multiple access with collision avoidance (CSMA/CA) and time division multiple access (TDMA).

This paper is organized as follows: A brief description of the multi-quadrotor dynamics and control over perfect communication links is introduced in section II. In Section III, the multi-quadrotor average consensus over wireless network analysis is presented. A stability analysis to improve the convergence of the consensus is proposed in section IV. Simulation results are illustrated in section V. And finally, conclusion and future work are discussed in section VI 


\section{Multi-QuAdrotor CONSEnsus}

\section{A. Quadrotor dynamic model and control}

Let us consider a group of $N$-quadrotor helicopters with the following dynamical model [25], [26]:

$$
\begin{aligned}
\ddot{x}_{i} & =-\mathbf{F}_{i} \sin \left(\theta_{i}\right) \\
\ddot{y}_{i} & =\mathbf{F}_{i} \cos \left(\theta_{i}\right) \sin \left(\phi_{i}\right) \\
\ddot{z}_{i} & =\mathbf{F}_{i} \cos \left(\theta_{i}\right) \cos \left(\phi_{i}\right)-1 \\
\ddot{\phi}_{i} & =\tau_{\phi, i} \\
\ddot{\theta}_{i} & =\tau_{\theta, i} \\
\ddot{\psi}_{i} & =\tau_{\psi, i}
\end{aligned}
$$

where $\mathbf{F}_{i}$ is the thrust force vector, $\left(x_{i}, y_{i}, z_{i}\right)$ and $\left(\phi_{i}, \theta_{i}, \psi_{i}\right)$ represent the position and orientation of the $i^{t h}$-quadrotor.

It was proved in [25] that the following control strategy stabilize the previous system

$$
\begin{aligned}
\mathbf{F}_{i}= & \frac{-k_{1, i} \dot{z}-k_{2, i}\left(z_{i}-z_{i}^{d}\right)+1}{\cos \left(\phi_{i}\right) \cos \left(\theta_{i}\right)} \\
\tau_{\psi, i}= & -k_{3, i} \dot{\psi}_{i}-k_{4, i}\left(\psi_{i}-\psi_{i}^{d}\right) \\
\tau_{\theta, i}= & -\sigma_{4}\left(\dot{\theta}_{i}+\sigma_{3}\left(\dot{\theta}_{i}+\theta_{i}+\sigma_{2}\left(\dot{\theta}_{i}+2 \theta_{i}\right.\right.\right. \\
& \left.\left.\left.-\dot{x}_{i}+\sigma_{1}\left(\dot{\theta}_{i}+3 \theta_{i}-3 \dot{x}_{i}-x_{i}\right)\right)\right)\right) \\
\tau_{\phi, i}= & -\sigma_{4}\left(\dot{\phi}_{i}+\sigma_{3}\left(\dot{\phi}_{i}+\phi_{i}+\sigma_{2}\left(\dot{\phi}_{i}+2 \phi_{i}+\dot{y}_{i}\right.\right.\right. \\
& \left.\left.\left.+\sigma_{1}\left(\dot{\phi}_{i}+3 \phi_{i}+3 \dot{y}_{i}+y\right)\right)\right)\right)
\end{aligned}
$$

where $k_{1, i}, k_{2, i}, k_{3, i}$ and $k_{4, i}$ are positive constant; $z^{d}$ and $\psi^{d}$ are the desired altitude and heading for the $i^{t h}$-quadrotor, respectively. Observe that the nonlinear control laws (7)-(10) guarantee the stabilization of the $i^{t h}$-quadrotor in closed loop system such that

$$
\begin{aligned}
& \lim _{t \rightarrow \infty} z_{i}=z^{d} \\
& \lim _{t \rightarrow \infty} \psi_{i}=\psi^{d} \\
& \lim _{t \rightarrow \infty} x_{i}=0 \\
& \lim _{t \rightarrow \infty} y_{i}=0
\end{aligned}
$$

\section{B. From individual to collective behavior}

In order to model the interactions among helicopters, a graph-based theoretical approach has been considered. [6] has established that the kinematic model of the $x$-position for a group of quadrotors can also be written as

$$
\dot{x}_{i}=\bar{u}_{i} \quad \forall i=1, . ., n ;
$$

with leader-based multi-quadrotor consensus achieved using the following algorithm

$$
\bar{u}_{i}=-\sum_{j \in \mathcal{N}_{i}}\left(x_{i}-x_{j}\right)
$$

where $\mathcal{N}_{i}$ is the set of vehicles transmitting their information to the quadrotor $i$. Noticed that (11) ensures the consensus agreement in the sense of $\lim _{t \rightarrow \infty}\left|x_{i}-x_{j}\right|=0$.

Hence, the position consensus among quadrotors yields

$$
\dot{\mathrm{x}}=-\mathcal{L} \mathbf{x}
$$

where $\mathcal{L}$ is the Laplacian matrix of the information exchange graph, more details see [9], [26].

Therefore, the controllers (7)-(10) can be improved to the case of multi-quadrotor consensus with the form

$$
\begin{aligned}
\mathbf{F}_{i}= & \frac{-a_{1, i} \dot{z}-a_{2, i}\left(\sum_{j \in \mathcal{N}_{i}}\left(z_{i}-z_{j}\right)-z^{d}\right)+1}{\cos \left(\phi_{i}\right) \cos \left(\theta_{i}\right)} \\
\tau_{\psi, i}= & -a_{3, i} \dot{\psi}_{i}-a_{4, i}\left(\sum_{j \in \mathcal{N}_{i}}\left(\psi_{i}-\psi_{j}\right)-\psi^{d}\right) \\
\tau_{\theta, i}= & -\sigma_{4}\left(\dot{\theta}_{i}+\sigma_{3}\left(\dot{\theta}_{i}+\theta_{i}+\sigma_{2}\left(\dot{\theta}_{i}+2 \theta_{i}-\dot{x}_{i}\right.\right.\right. \\
& \left.\left.+\sigma_{1}\left(\dot{\theta}_{i}+3 \theta_{i}-3 \dot{x}_{i}-\left(\sum_{j \in \mathcal{N}_{i}}\left(x_{i}-x_{j}\right)\right)\right)\right)\right) \\
\tau_{\phi, i}= & -\sigma_{4}\left(\dot{\phi}_{i}+\sigma_{3}\left(\dot{\phi}_{i}+\phi_{i}+\sigma_{2}\left(\dot{\phi}_{i}+2 \phi_{i}+\dot{y}_{i}\right.\right.\right. \\
& \left.\left.+\sigma_{1}\left(\dot{\phi}_{i}+3 \phi_{i}+3 \dot{y}_{i}-\left(\sum_{j \in \mathcal{N}_{i}}\left(y_{i}-y_{j}\right)\right)\right)\right)\right)
\end{aligned}
$$

which implies that

$$
\begin{aligned}
\lim _{t \rightarrow \infty}\left|\left(z_{j}-z_{i}\right)\right| & =z^{d} \\
\lim _{t \rightarrow \infty}\left|\left(\psi_{j}-\psi_{i}\right)\right| & =\psi_{i}^{d} \\
\lim _{t \rightarrow \infty}\left|\left(x_{j}-x_{i}\right)\right| & =0 \\
\lim _{t \rightarrow \infty}\left|\left(y_{j}-y_{i}\right)\right| & =0
\end{aligned}
$$

In order to illustrate the performance of the previous control strategy, simulations were carried, over the $x$-axis, out considering a 10-quadrotor platoon over a perfect communication network (no delay, no packet loss) with cyclic topology. The initial conditions are $x_{i}(0)=i, \forall i=$ $0,1, \ldots, 9$

Figure 1 shows the consensus response. Observe in this figure that the convergence time is small and the solution of the average consensus over the $x$-axis is the average of the initial conditions, i.e., the platoon achieves consensus to 4.5. Thus, the previous nonlinear control laws guarantees the position synchronization of the quadrotor platoon, see [26].

In literature, several simulations using the previous (similar or different) controllers have been carried out in order to prove the stability of the consensus or of the flight formation trajectory, see [10], [11], [12], [13]. Most of the assumptions in these works are considering wireless and perfect communication between the vehicles. In addition, the main results in flight formation are in general illustrated only in simulations.

As it is known, in a real multi-vehicle flight formation system, each aircraft collects information from its sensors and then exchange its information, employing wireless communication, with other autonomous vehicles in the network. Packet delay and packet loss in wireless becomes a major issue of study that must be taken into account when stabilizing multi-aerial vehicles. The goal of this work is to prove, in simulations, the impact of the wireless network communication in a multi-vehicle consensus. 


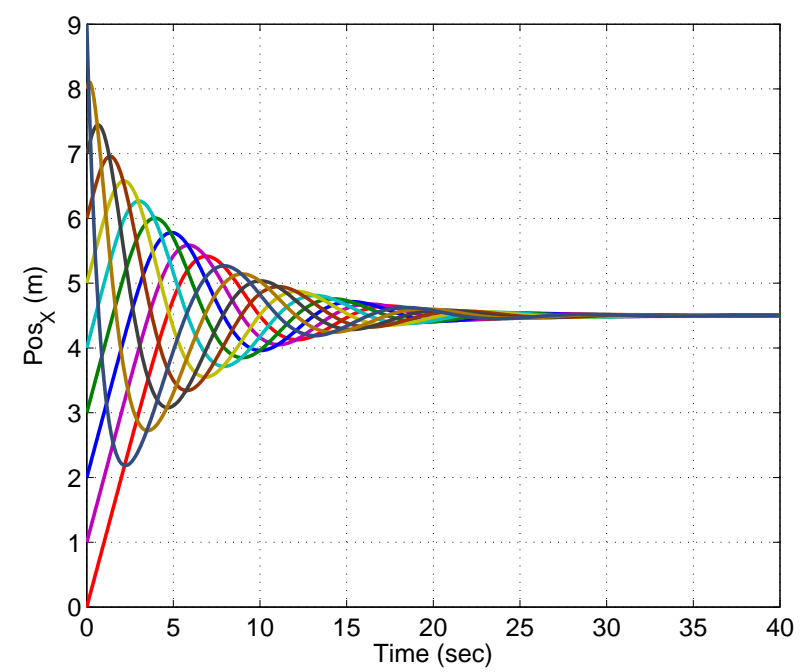

Fig. 1. Multiple quadrotor consensus using perfect communication

\section{Multi-Agent Consensus over Wireless NETWORKS}

Let us consider the case of a $\mathrm{N}$-quadrotor formation flying over a wireless communication channel. From the automatic control point of view, the key factors when using wireless channels are: end-2-end time delay, packet dropout rate, network connectivity and noise. Let us assume that the mobility of the agents does not affect the network connectivity and neglect the noise from sensors. Then, we focus our attention on the packet dropout rate and the end-2-end time delay problems. It has been shown in the communications literature [30], [31] that both packet dropout rate and the end-2-end time delay are determined by the Medium Access Control protocols. Our study considers two common technologies for wireless communications: CSMA/CA used in IEEE802.11 and TDMA used in GSM.

\section{A. CSMA/CA}

This is a distributed random access algorithm used in many standards such as Wifi IEEE802.11. This scheme uses a carrier sense multiple access with collision avoidance (CSMA/CA) mechanism for resolving the problem of access to the communication medium. This implies that when a node detects a collision, it stops transmitting and waits for a random time before retransmitting. More precisely, the protocol CSMA/CA works as follows [29]:

1) a carrier sensing scheme is used

2) a data station that intends to transmit sends a jam signal,

3) after waiting a sufficient time for all stations to receive the jam signal, the data station transmits a frame

4) while transmitting, if the data station detects a jam signal from another station, it stops transmitting for a random time and then tries again.

We distinguish two variants: with and without channel reservation. In CSMA/CA with channel reservation, the transmitter first transmits a RTS (Request to Send) for channel reservation, and waits for receiving a CTS (Clear To Send) from the destination before starting to send the data frame.

CSMA/CA is not suitable for real time communications, since it does not guarantee a upper bound for the delay before sending a data frame.

\section{B. TDMA}

This is a time slotted scheme used in many standards such as GSM. Accordingly with [29], TDMA (Time Division Multiple Access) is a collision-free multiple access technique whereby users share a transmission medium by being assigned and using (one at a time) time slots assigned previously.

TDMA is more suitable for real time communications, since it guarantees a upper bound delay before transmitting a data frame.

\section{Network Analysis}

To evaluate the performance of CSMA/CA and TDMA protocols on the quadrotor consensus problem, extensive simulations have been run using the network simulator NS2. As it is shown in Figures 2 and 3, it is clear that the packet drops for TDMA protocol are almost null. We attribute the packet drops shown in Figure 2 to the synchronization phase during the simulation initialization. Unlike TDMA, CSMA/CA shows a higher rate of packet drops due to its random broadcasting nature, see Figure 3 .

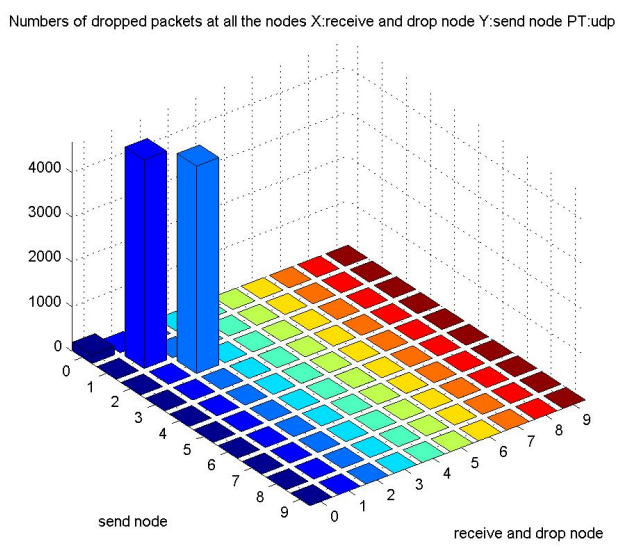

Fig. 2. Packet drop using TDMA

Now, let us analyze the average end-2-end delay which gives an insight of the expected time delay on multiquadrotor systems. It is known that in real-time applications, after a large time delay, data may become useless. Therefore it is important to analyze the performance of MAC protocols such that the multi-quadrotor system will undergo the minimal time delay. On one hand, since TDMA is a time division multiple access technique to access the transmission medium, it is almost intuitively that the time delay, should show an 


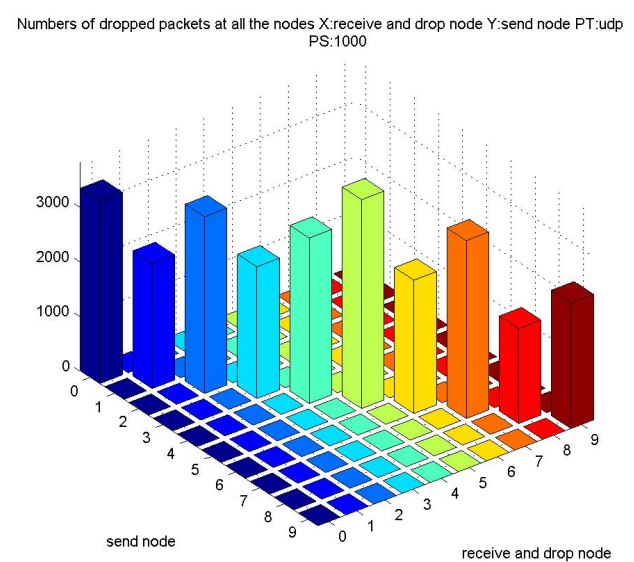

Fig. 3. Packet drop using CSMA/CA

almost constant rate. This can be confirmed by observing Figure 4. On the other hand, taking into account the packet drop rate at each quadrotor, it is expected that CSMA/CA would show a variable time delay and the maximum time delay is much higher than TDMA. The evolution of the average end-2-end time delay over time for the CSMA/CA protocol is shown in Figure 5.

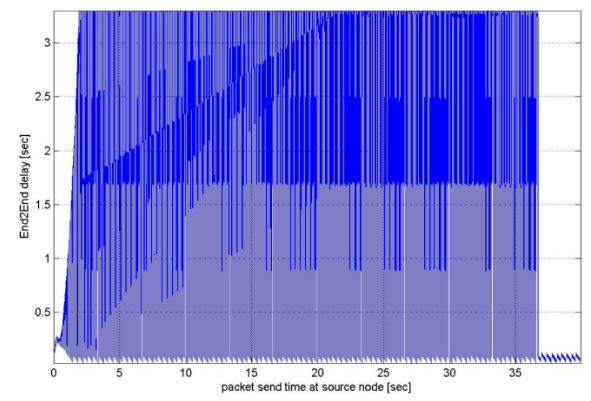

Fig. 4. Packet time delay using TDMA

\section{Quadrotor CONSENSUS OVER Wireless NETWORKS}

In this section, we can introduce a Network-aware average consensus control for a multi-quadrotor system over a wireless network. By taking into account the phenomena discussed above, the packet dropout process will be considered as a source of time delays in the wireless network. As shown in [30], the end-2-end time delay $\tau$ is given by the difference $\tau_{d s t}-\tau_{s r c}$ which in turns depends on the preprocessing time $\tau_{\text {pre }}$, wait time $\tau_{\text {wait }}$, transmission time $\tau_{t x}$ and the post processing time $\tau_{\text {post }}$. Since the nearest neighbor approach assumes that each vehicle communicates only with immediate neighbors that are in its radio range, and assuming that a packet loss implies the re-transmission of the data that has been lost, we consider the packet dropout process, as part of the transmission time

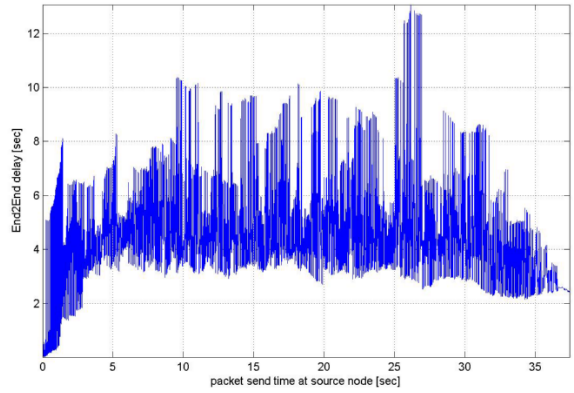

Fig. 5. Packet time delay using CSMA/CA

delay which has been defined as the frame time $\tau_{\text {frame }}$ and the propagation time $\tau_{\text {prop }}$, more details see [30].

Then, we propose to use the following multi-quadrotor consensus control as in [32]

$$
\bar{u}_{i}(t)=-\sum_{j \in \mathcal{N}_{i}}\left(x_{i}-x_{j}\left(t-\tau_{j i}\right)\right)
$$

Using the control law (17) instead of (11), the quadrotor consensus control is given as

$$
\begin{aligned}
\mathbf{F}_{i}= & \frac{-k_{1, i} \dot{z}_{i}-k_{2, i}\left(\sum_{j \in \mathcal{N}_{i}}\left(z_{j}\left(t-\tau_{j i}\right)-z_{i}\right)-z^{d}\right)+1}{\cos \left(\phi_{i}\right) \cos \left(\theta_{i}\right)} \\
\tau_{\psi, i}= & -k_{3, i} \dot{\psi}_{i}-k_{4, i}\left(\sum_{j \in \mathcal{N}_{i}}\left(\psi_{j}\left(t-\tau_{j i}\right)-\psi_{i}\right)-\psi^{d}\right) \\
\tau_{\theta, i}= & -\sigma_{4}\left(\dot{\theta}_{i}+\sigma_{3}\left(\dot{\theta}_{i}+\theta_{i}+\sigma_{2}\left(\dot{\theta}_{i}+2 \theta_{i}-\dot{x}_{i}\right.\right.\right. \\
& \left.\left.\left.+\sigma_{1}\left(\dot{\theta}_{i}+3 \theta_{i}-3 \dot{x}_{i}-\sum_{j \in \mathcal{N}_{i}}\left(x_{j}\left(t-\tau_{j i}\right)-x_{i}\right)\right)\right)\right)\right) \\
\tau_{\phi, i}= & -\sigma_{4}\left(\dot{\phi}_{i}+\sigma_{3}\left(\dot{\phi}_{i}+\phi_{i}+\sigma_{2}\left(\dot{\phi}_{i}+2 \phi_{i}+\dot{y}_{i}\right.\right.\right. \\
& \left.\left.\left.+\sigma_{1}\left(\dot{\phi}_{i}+3 \phi_{i}+3 \dot{y}_{i}-\sum_{j \in \mathcal{N}_{i}}\left(y_{j}\left(t-\tau_{j i}\right)-y_{i}\right)\right)\right)\right)\right)
\end{aligned}
$$

Let us consider the Lyapunov function as in [32]

$$
V=\sum_{i=1}^{N} \sum_{j \in N} \int_{t-\tau_{j i}}^{t} x_{j}^{T}(\chi) x_{j}(\chi) d \chi+2\left(V_{1}+\ldots+V_{N}\right)
$$

where $V_{i}$ is the storage function for each quadrotor vehicle in the platoon. Differentiating (22),

$\dot{V}=-2 \sum_{i=1}^{N} S_{i}\left(x_{i}\right)-\sum_{i=1}^{N} \sum_{j \in N_{i}}\left(x_{j}\left(t-\tau_{j i}\right)-x_{i}\right)^{T}\left(x_{j}\left(t-\tau_{j i}\right)-x_{i}\right)$

From (23) we have that

$$
\lim _{t \rightarrow \infty}\left|\left(x_{j}\left(t-\tau_{j i}\right)-x_{i}\right)\right|=0
$$

From (24), it is clear that the solution of the average consensus to the average of the initial conditions depends on the network medium access algorithm, which in turns determines the values of the $\tau_{j i}$ for each quadrotor. Figure 6 shows the performance of the average consensus considering 
$\tau_{j i}=\tau_{k j}$. It can be observed that the average consensus is the same than the one for the perfect communication case. The difference is that the convergence time for the equal time delay case is larger than for the perfect communication case. Figure 7 shows the performance of the average consensus

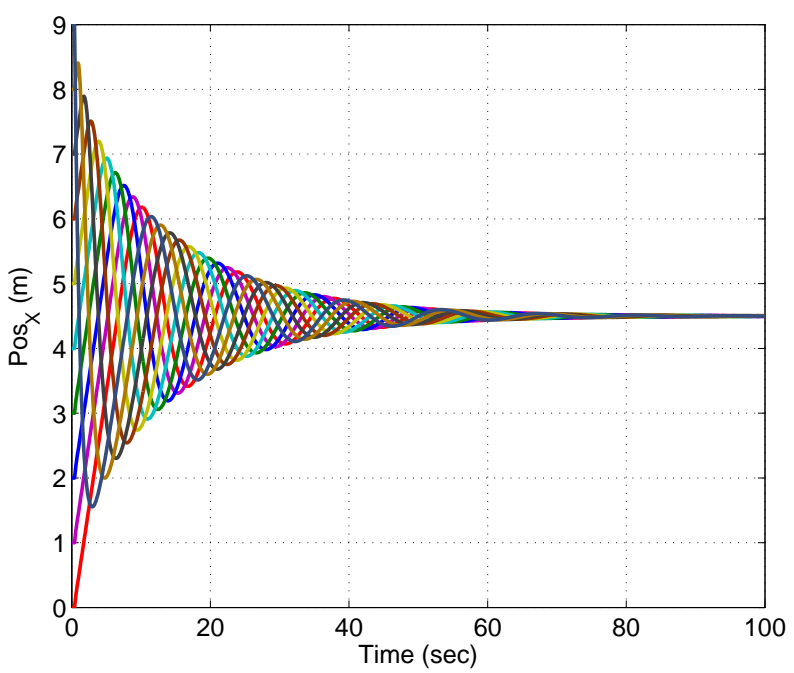

Fig. 6. Multiple quadrotor consensus with $\tau_{j i}=\tau_{k j}$

considering $\tau_{j i} \neq \tau_{k j}$. It can be observed that the solution of average consensus is different from the previous two cases: perfect communication case and equal time delays case.

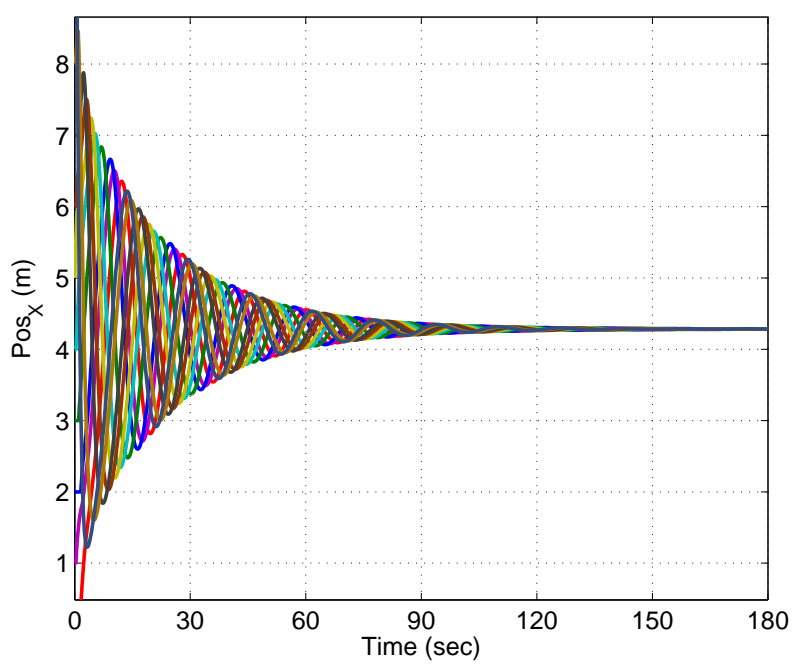

Fig. 7. Multiple quadrotor with $\tau_{j i} \neq \tau_{k j}$

\section{Simulation Results}

Now, lets take a look at the average consensus performance using a wireless network. The network scenario consisted of 10-quadrotors sharing information over a cyclic topology. A quadrotor application was developed, a UDP transport protocol was modified to exchange information with the quadrotor application and the MAC layer as shown in Figure 8. The following MAC algorithms: CSMA/CA and TDMA

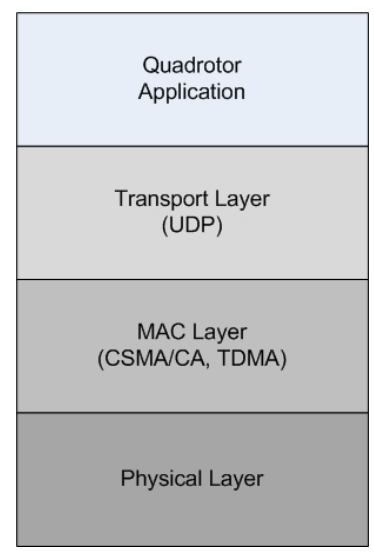

Fig. 8. Multiple quadrotor wireless network stack

were analyzed using the network simulator NS2. Figures 9 and 10 show the performance of the MAC protocols CSMA/CA and TDMA respectively.

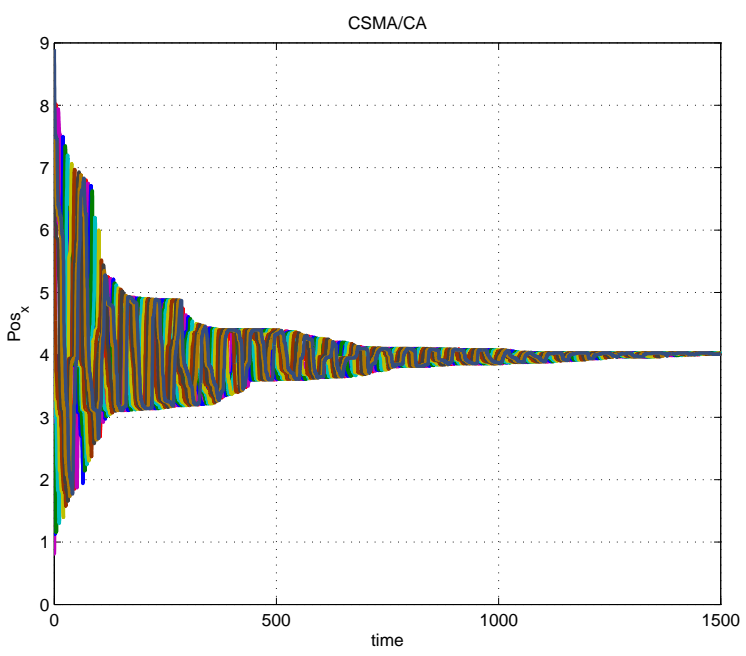

Fig. 9. Multi-agent consensus using IEEE 802.11

From Figures 9 and 10, it is possible to observe that the solution for the average consensus as well as its convergence time are affected by the network media access control algorithm. It can be concluded that the average consensus using a CSMA/CA protocol presents a larger convergence time due to both packet drops and time delay in the end-2end transmission among quadrotors. Also, due to the fact that the access to the transmission medium is assigned randomly, it is evident that some quadrotors will transmit their positions to their neighbors before their counterparts. This implies that the difference between any two $\tau_{j i}$ can be large enough such that some quadrotors will evolve faster than the others. Then, the quadrotor with the smallest $\tau_{j i}$ will 


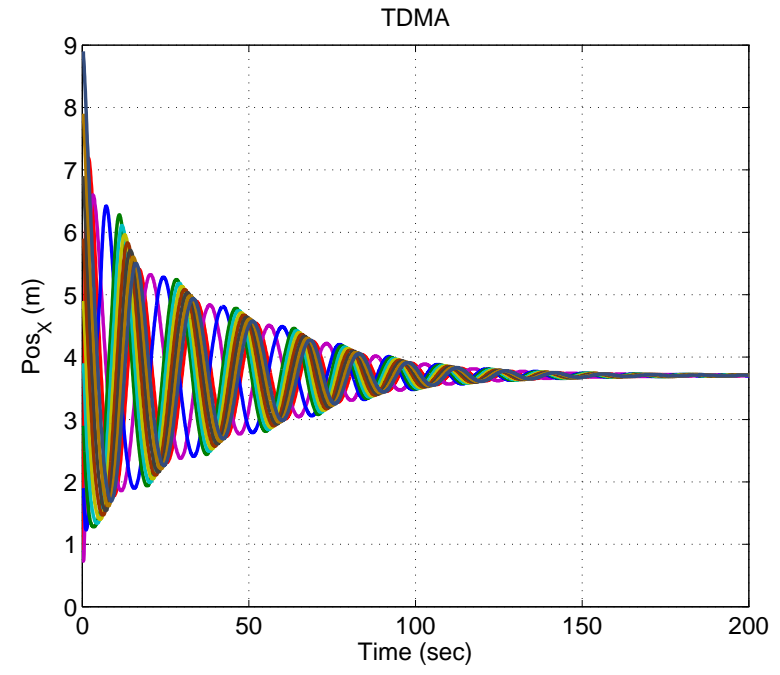

Fig. 10. Multi-agent consensus using TDMA

update its position at a highest rate than the others. Figure 11 shows the performance of the average consensus for a small platoon of 4 quadrotors using different medium access control algorithms such as: CSMA/CA, TDMA, constant time delay $\left(\tau_{j i}>0\right)$, and perfect communication.

The simulation results are summarized in Table I

TABLE I

PERFORMANCE COMPARISON

\begin{tabular}{lccc}
\hline MAC & $\begin{array}{c}\text { Packet Drop } \\
\text { Algorithm }\end{array}$ & $\begin{array}{c}\text { Max E-2-E Time } \\
\text { Delay }\end{array}$ & $\begin{array}{c}\text { Convergence } \\
\text { Time }\end{array}$ \\
\hline CSMA/CA & $\sim 8.3 \%$ & $\sim 12$ & $\sim 1200$ \\
TDMA & $\sim 7.2 \%$ & $\sim 3.2$ & $\sim 200$ \\
Perfect Comm. & 0 & 0 & $\sim 40$ \\
\hline
\end{tabular}

Figure 12 shows the performance of the average consensus for the heading subsystem of a 2-quadrotor system using CSMA/CA.

\section{CONCLUSiOnS AND Future Work}

A nonlinear control based on nested saturations and a single integrator consensus control considering time delay for flight formation of mini rotorcraft was developed. We remark that TDMA is more suitable for real time communications than CSMA/CA, since it guarantees a upper bound delay before transmitting a data frame. CSMA/CA is less suitable suitable for real time communications, since it does not guarantee an upper bound for the delay before sending a data frame. Extensive simulations were run in order to show the performance of the developed control scheme. Future work in this area includes experimental tests on mini rotorcraft using real-time embedded control systems and to develop an optimized medium access control algorithm for mobile robot systems.

\section{REFERENCES}

[1] V. Gazi and Kevin M. Passino, "Stability Analysis of Swarms", IEEE Trans. on Autom. Control, Vol. 48, No. 4, pp.692-697, 2003.
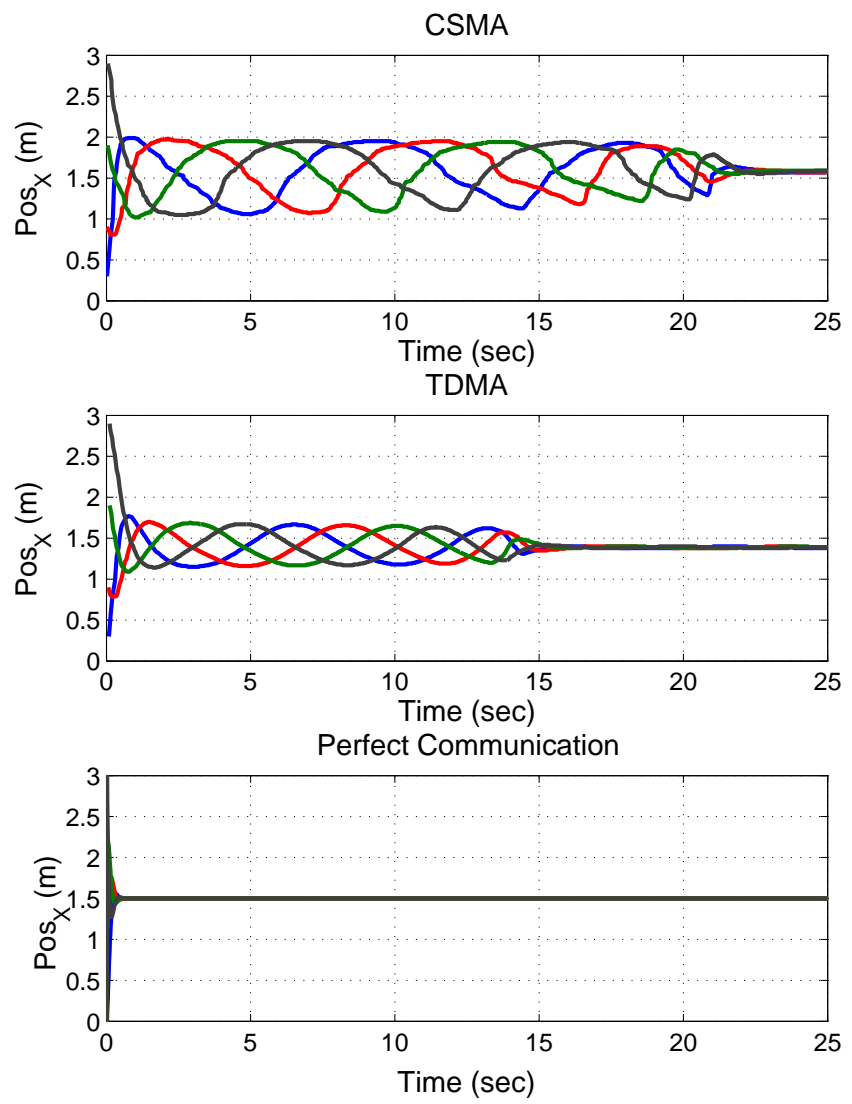

Fig. 11. Comparison of convergence time for different MAC algorithms

[2] V. Gazi, "Stability of an Asynchronous Swarm With Time-Dependent Communication Links", IEEE Trans. on Systems, Man, and Cybernetics, Vol. 38, No. 1, pp.267 - 274, 2008.

[3] R. Olfati-Saber, "Flocking for Multi-Agent Dynamic Systems: Algorithms and Theory", IEEE Trans. on Autom. Control, 2006.

[4] H.G. Tanner, A. Jadbabaie and G.J. Pappas, "Stable Flocking of Mobile Agents, Part I: Fixed Topology", Proceedings of the 42nd IEEE Conference on Decision and Control, 2003.

[5] H.G. Tanner, A. Jadbabaie and G.J. Pappas, "Stable Flocking of Mobile Agents, Part II: Dynamic Topology", Proceedings of the 42nd IEEE Conference on Decision and Control, 2003.

[6] D. Lee and Mark W Spong, "Stable Flocking of Multiple Inertial Agents on Balanced Graphs", IEEE Trans. on Autom. Control, Vol. 52, No. 4, pp. 681-686, 2007.

[7] W. Ren, "Consensus Seeking in Multi-vehicule Systems with a Time Varying Reference State", Proceedings of the American Control Conference, 2007.

[8] M. Porfiri, D. G. Roberson and Daniel J Stilwell, "Tracking and formation control of multiple autonomous agents : A two-level consensus approach", Automatica, Vol. 43, pp. 1318 - 1328, 2007.

[9] J.A. Guerrero, G. Romero and R. Lozano, "Robust Consensus Tracking of Leader-Based Multi-Agent Systems", Proceedings of the IEEE American Control Conference (ACC), 2010.

[10] J.A. Fax and R. Murray, "Information Flow and Cooperative Control on Vehicle Formations", IEEE Trans. on Autom. Control, Vol. 49, No. 9, pp. 692-697, 2004.

[11] G. Lafferriere, A. Williams, J. Caughman and J. J. P. Veerman, "Decentralized control of vehicle formations", Systems \& Control Letters, Vol. 54, pp. 899 - 910, 2005.

[12] W. B. Dunbar and Richard M Murray, "Distributed receding horizon control for multi-vehicle formation", Automatica, Vol. 42, pp. 549 $558,2006$. 

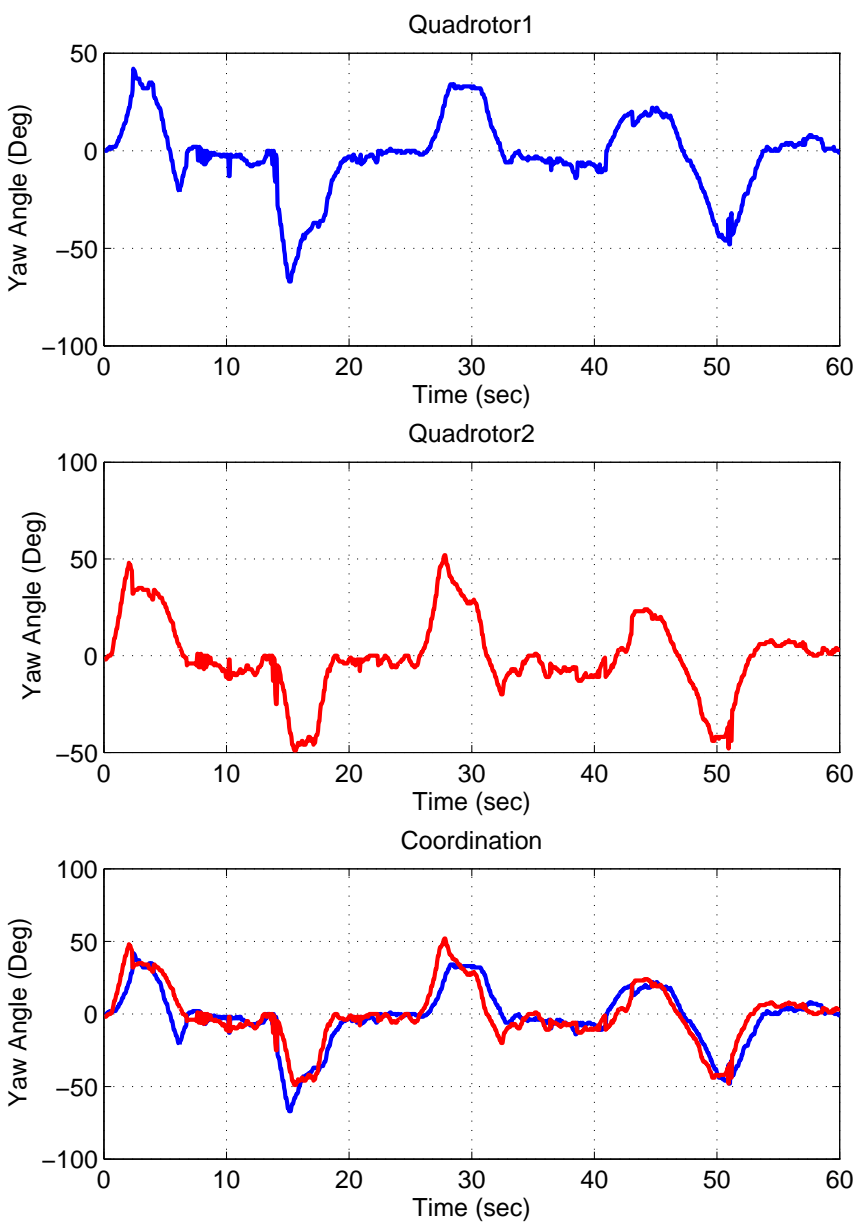

Fig. 12. Average consensus for the yaw subsystem. An external reference was given to the system in order to show the delay over the wireless network

[13] X. Chen and A. Serrani, "ISS-Based Robust Leader/Follower Trailing Control", LNCIS 336 Group Coordination and Cooperative Control, Springer-Verlag, 2006.

[14] R. Kristiansen, A. Loria, A. Chaillet and P.J. Nicklasson, "Output Feedback Control of Relative Translation in a Leader-Follower Spacecraft Formation", LNCIS 336 Group Coordination and Cooperative Control, Springer-Verlag, 2006.

[15] N.E. Leonard and E. Fiorelli, "Virtual Leaders, Artificial Potentials and Coordinated Control of Groups", Proceedings of the 40th IEEE Conf. on Decision and Control, 2001.

[16] R.W. Beard, J. Lawton and F.Y. Hadaegh, "A Coordination Architecture for Spacecraft Formation Control", IEEE Transactions on Control Systems Technology, Vol. 9, No. 6, 2001.

[17] F. Arrichello, S. Chiaverini and T.I. Fossen, "Formation Control of Marine Vessels using the Null-Space-Based Behavioral Control", LNCIS 336 Group Coordination and Cooperative Control, SpringerVerlag, 2006.

[18] T. Balch and R.C. Arkin, "Behavior-based Formation Control for multirobot teams", IEEE Transactions on Robotics and Automation, Vol. 14, No. 6, 1998.

[19] A. Sahai, "The necessity and sufficiency of anytime capacity for control over noisy cummincation link", Proceedings of the 43rd IEEE Conf. on Decision and Control, 2004.

[20] S. Takikonda and S. Mitter, "Control over Noisy Channels", IEEE Trans. on Autom. Control, Vol. 49, No. 7, pp. 1196-1201, 2004.

[21] W. Lin, L. ZhiXin, "Robust Consensus of Multi-Agent Systems with
Noise", Sci China Ser F-Inf Sci., Vol. 52, No.5, pp. 824-834, 2009.

[22] W. Zhang, Z. Wang and Y. Gou, "Robust Consensus for Uncertain Multi-agent Systems on Directed Communication Topologies", Proceedings of the 49th IEEE Conference of Decision and Control, Baltimore, 2010.

[23] S. Hu, and W-Y. Yan, "Stability Robustness of Networked Control Systems with Respect to Packet Loss", Automatica, Vol. 43, pp. 1243 $1248,2007$.

[24] Q. Ling, and M.D. Lemmon, "Soft Real-Time Scheduling of Networked Control Systems with Dropouts Governed by a Markov Chain", Proceedings of the IEEE American Control Conference, Vol 6, pp. 4845-4850, 2003.

[25] R. Lozano, "Unmanned Aerial Vehicles Embedded Control ", WileyISTE, 2010.

[26] J.A. Guerrero, I. Fantoni, S. Salazar, R. Lozano, Flight Formation of Multiple Rotorcrafts., in IEEE International Conference on Robotics and Automation (ICRA 2010), Alaska, Mai 2010.

[27] P. Castillo, R. Lozano and A. Dzul, "Stabilization of a mini-rotorcraft having four rotors", in IEEE Control Systems Magazine, Vol.25, No. 6, pp. 45-55, Dec. 2005

[28] C. Godsil and G.F. Royle, Algebraic Graph Theory, Springer, 2001.

[29] Alliance for Telecommunications Industry Solutions, Telecom Glossary, 2007.

[30] F-L. Lian, J.R. Moyne and D.M. Tilbury "Performance Evaluation of Control Networks: Ethernet, ControlNet and DeviceNet" in IEEE Control Systems Magazine, Vol. 21, No. 1, pp. 66-83, Feb. 2001.

[31] X. Liu, A. Goldsmith, Wireless medium access control in networked control systems, in IEEE American Control Conference (ACC 2004), Boston, USA, July 2004

[32] N. Chopra, Output Synchronization of Mechanical Systems, UIUC PhD Thesis, 2006. 